\title{
SELF-TUNING WEIGHTING FACTOR TO DECOUPLING CONTROL FOR INCUBATOR SYSTEM
}

\author{
M. A. Zermani ${ }^{1}$, E. Feki ${ }^{2}$ and A. Mami $^{3}$ \\ ${ }^{1}$ Department of Electric Engineering, National School of Engineering of Tunis \\ Aymen_netevoila.fr \\ ${ }^{2,3}$ Department of Physics, Faculty of Science, Mathematics, Physics and Natural of Tunis, \\ Campus Universities 2092 El Manar,Tunisia
}

\begin{abstract}
This paper is focused on a design of an algorithm for decoupling multivariable systems based on generalized predictive control (DGPC) and its application to a control of an incubator system. Proposed control algorithm uses adaptive error weighting factor in cost function in order to reduce coupling between control loops. Two techniques are presented and compared for predictive control of TITO (Two-Input, Two-Output) processes. According to the first technique the weighting factor is tuned in synchronization to a reference signal change. According to the second method, the weighting factor is set dependent on the actual control error. The algorithm is applied to incubator climate control; obtained simulation results show its efficiency.
\end{abstract}

\section{KEYWORDS}

Incubator Process, Decoupling Predictive Control, Adaptive Error Weighting Factor

\section{INTRODUCTION}

Many industrial processes have strong cross coupling between control loops. Mostly Proportional Integral Differential (PID) is the simplest solution to control these systems. But these controllers do not offer satisfactory control because of interactions. Also the tuning of PID is complicated when they are used in MIMO systems. Generalized predictive control appears to be attractive solution in multivariable process control [5,10]. However, these controllers cannot solve problems with strong interaction. In that case, decoupling can perfectly achieved by adding interaction compensators to controllers [2]. But in some systems because of improper delay structure or model mismatch, interaction compensators cannot applicable [3]. Therefore, a practical method for decoupling is required and compared. The first technique, the weighting factor is tuned in synchronization to a reference signal change. According to the second method, the weighting factor is set dependent on the actual control error. Most of the industrial systems are infected with disturbance; the presence of this term can complicate the tuning of weighting factor. To solve this problem we used a first-order filter. The aim of this contribution is an implementation of the GPC control with self-tuning of the weighting factor including constraints of the manipulated variable for control of the neonatal incubator. 
The incubator process is used to produce healthful micro-environment in order to reduce new born heat loss by controlling temperature inside incubator. Temperature is one of the most important factors that need to be maintained with a minimum variation. But only temperature control is not sufficient to provide comfortable environment. Also, the relative humidity control is very important to reduce the new born heat loss $[8,9]$. For these reasons, we have developed an active humidification system based on a nebulizer. Therefore, this process becomes a TITO system (Two Input-Two-Output). In fact, the process has time-varying parameters model, depending on the external temperature and on the load under treat. Also, couplings between temperature and humidity are very important that should be reduced.

The paper is structured as follows. The section 2 is devoted to present decentralized generalized predictive control with constraint. In section 3, we present the first technique of decoupling based on synchronization of the weighting factor with a reference signal change. In Section 4, we propose the second method of decoupling which the weighting factor is set dependent on the actual control error. In Section 5 experimental setup and computer simulations are conducted to review the feasibility and effectiveness of the proposed method, to decrease interaction cross coupling between controlled variables (temperature and humidity) of incubator system.

\section{THEORY}

\subsection{Decentralized Control}

Decentralized control (Benhammou 1988, Lunze 1992, Gagnon 1999) is based on several local controllers, instead of a single controller to control a multivariable process (MIMO). Each of these controllers is responsible for controlling a single loop. The decentralized control scheme for a rnultivariable process is illustrated in Fig 1.

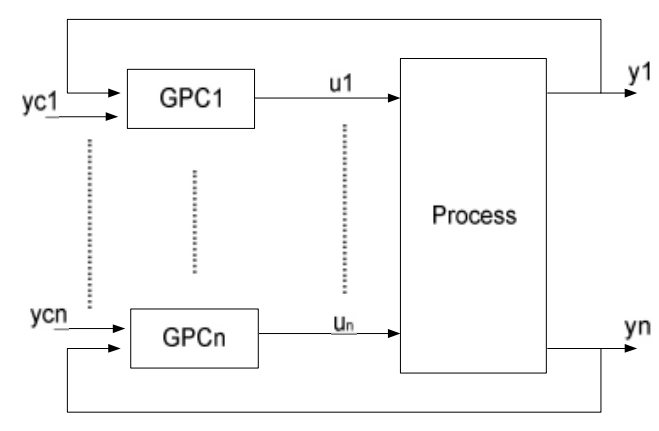

Figure 1. Structure of decentralized control (or distributed)

This decentralized approach provides some advantages, both theoretical and practical very interesting to quote: Structure is easier to achieve (very simple and fast to implement it in industry).This approach is both flexible (the failure of a party system can be treated without affecting the rest, maintenance is also more easily). It is feasible to specify different performance and use of different sampling periods for each particular loop. Removal or addition of a control loop or manual mode setting does not affect the overall stability of system (other loops continue to be compensated). For these reasons, we take into count this structure Fig. 1, for the conception of the decoupling predictive control. 


\subsection{Process Model}

The synthesis of the generalized predictive controller (GPC) suggested by (Clarke et al, 1987; Clarke, 1988) [5,6]. This method was used successfully in industrial applications of various forms (Richalet et al,1987; Richalet et al,2009) [11,12]. The approach of generalized predictive control is based on a dynamic model of type ARIMAX (Auto Regressive Integrated Moving Average with eXternal inputs), given by:

$$
A_{i}\left(z^{-1}\right) y_{i}(k)=z^{-d_{i}} B_{i}\left(z^{-1}\right) u_{i}(k-1)+C_{i}\left(z^{-1}\right) \frac{e_{i}(k)}{\Delta\left(z^{-1}\right)} \text {. }
$$

Where $i$ is the number system, $y_{i}(k)$ is the system output, $u_{i}(k)$ is the system input, $e_{i}(k)$ is the uncorrelated random sequence, $\Delta\left(z^{-1}\right)=1-z^{-1}$ corresponds to an integral action. Its presence in the direct channel allows a zero error in steady state value. $A_{i}\left(z^{-1}\right), B_{i}\left(z^{-1}\right)$ and $C_{i}\left(z^{-1}\right)$ are polynomials.

$$
\begin{aligned}
& A_{i}\left(z^{-1}\right)=1+a_{1} z^{-1}+a_{2} z^{-2}+\cdots+a_{n a i} z^{-n a_{i}}, \\
& B_{i}\left(z^{-1}\right)=b_{0}+b_{1} z^{-1}+b_{2} z^{-2}+\cdots+b_{n b i} z^{-n b_{i}} \\
& C_{i}\left(z^{-1}\right)=1+c_{1} z^{-1}+c_{2} z^{-2}+\cdots+c_{n c i} z^{-n c i}
\end{aligned}
$$

With $n a_{i}, n b_{i}$ et $n c_{i}$ indicate the respective order of these polynomials.

The generalized predictive control based on the minimization of a quadratic criterion on a sliding horizon, which involves a term related to the difference between the predicted output sequence and the sequence of future control [4].

The criterion is given by:

$$
J_{i}=\lambda_{3 i} \sum_{=N_{i}}^{H P_{i}}\left[y_{C_{i}}(k+t)-\hat{y}_{i}(k+t)\right]^{2}+\lambda_{u i} \sum_{i=1}^{N_{c_{i}}} \Delta u_{i}^{2}(k+t-1)
$$

with $\hat{y}_{i}(k)$ is the output value predicted at time $\mathrm{k}, y_{C_{i}}(k)$ is the set points values at time $\mathrm{k}, \Delta u_{i}(k)$ is the increment of control at time $\mathrm{k}, N_{i}$ is the minimum prediction horizon, $H P_{i}$ is the maximum prediction horizon, $N_{c_{i}}$ is the control horizon, $\lambda_{u i}$ is the control weighting factor and $\lambda_{y i}$ is the error weighting factor.

\subsection{Prediction of the System Output}

Consider the output expressed by (1) the output at time instant $(k+t)$ will be:

$$
y_{i}(k+t)=\frac{B_{i}\left(z^{-1}\right)}{A\left(z^{-1}\right)} u_{i}\left(k+t-d_{i}-1\right)+\frac{C_{i}\left(z^{-1}\right)}{A\left(z^{-1}\right) \Delta\left(z^{-1}\right)} e_{i}(k+t)
$$

By applying the Euclidean algorithm on the second term of (4) we get

$$
\frac{C_{i}\left(z^{-1}\right)}{A_{i}\left(z^{-1}\right) \Delta\left(z^{-1}\right)}=L_{t}\left(z^{-1}\right)+z^{-t} \frac{G_{t}\left(z^{-1}\right)}{A_{i}\left(z^{-1}\right) \Delta\left(z^{-1}\right)}
$$

After using (4) and (5), we assume that the term related to the disturbance is zero, the optimal predictor of the output is:

$$
\hat{y}_{i}(k+t)=\frac{L_{i}\left(z^{-1}\right) B_{i}\left(z^{-1}\right) \Delta\left(z^{-1}\right)}{C_{(}\left(z^{-1}\right)} u_{i}\left(k+t-d_{i}-1\right)+\frac{G\left(z^{-1}\right)}{C_{i}\left(z^{-1}\right)} y_{i}(k)
$$


International Journal of Information Technology, Control and Automation (IJITCA) Vol.2, No.3, July 2012

A second Diophantine equation decompose the predictor in two terms: a term based on the current output, old orders, the system output and a second term dependent on future orders.

$$
\frac{\sigma_{i}\left(z^{-1}\right)}{C_{i}\left(z^{-1}\right)}=H_{t}\left(z^{-1}\right)+z^{-t+d_{i}} \frac{R_{t}\left(z^{-1}\right)}{C_{i}\left(z^{-1}\right)}
$$

With:

$$
\sigma_{i}\left(z^{-1}\right)=L_{t}\left(z^{-1}\right) B_{i}\left(z^{-1}\right)
$$

The optimal predictor of the output is:

$$
\begin{aligned}
\hat{y}_{i}(k+t) & =H_{t}\left(z^{-1}\right) \Delta\left(z^{-1}\right) u_{i}\left(k+t-d_{i}-1\right)+ \\
& \frac{G_{t}\left(z^{-1}\right)}{C_{i}\left(z^{-1}\right)} y_{i}(k)+\frac{R_{t}\left(z^{-1}\right)}{C_{i}\left(z^{-1}\right)} \Delta\left(z^{-1}\right) u_{i}(k-1)
\end{aligned}
$$

Where $H_{t}\left(z^{-1}\right) G_{t}\left(z^{-1}\right) R_{t}\left(z^{-1}\right)$ and $L_{t}\left(z^{-1}\right)$ are polynomial solutions to the Diophantine equations [5]. The matrix formulation is represented by:

$$
\hat{Y}_{i}=\hat{H}_{i} \Delta U_{i}+\hat{G}_{i} Y_{i}^{*}+\hat{R}_{i} \Delta U_{i}^{*}
$$

The vector of the predicted outputs $\hat{Y}_{i}$ is the sum of the predicted forced $\hat{H}_{i} \Delta U_{i}$ and free responses $\hat{G}_{i} Y_{i}^{*}+\hat{R}_{i} \Delta U_{i}^{*}$

With:

$$
\begin{aligned}
& \hat{Y}_{i}=\left[\hat{y}_{i}(k+1 / k), \hat{y}_{i}(k+2 / k) \cdots \hat{y}_{i}\left(k+H P_{i} / k\right)\right]^{T}, \\
& Y_{i}^{*}=\left[y_{i}^{*}(k), y_{i}{ }^{*}(k-1) \cdots y_{i}^{*}\left(k-n a_{i}\right)\right]^{T}, \\
& \Delta U_{i}=\left[\Delta u_{i}(k) \cdots \Delta u_{i}\left(k+N_{C i}-1\right)\right]^{T}, \\
& \Delta U_{i}^{*}=\left[\Delta u_{i}^{*}(k-1) \cdots \Delta u_{i}^{*}(k-n b i-d i+1)\right]^{T}, \\
& \hat{G}_{i}=\left[G_{1+d_{i}}\left(z^{-1}\right) \cdots G_{H P_{i}+d_{i}}\left(z^{-1}\right)\right]^{T}, \\
& \hat{R}_{i}=\left[R_{1+d_{i}}\left(z^{-1}\right) \cdots R_{H P_{i}+d_{i}}\left(z^{-1}\right)\right]^{T}, \\
& \hat{H}_{i}=\left(\begin{array}{cccc}
h_{0} & 0 & \cdots & 0 \\
h_{1} & h_{0} & \cdots & 0 \\
\vdots & \vdots & \vdots & \vdots \\
h_{H P_{i}-d i-1} & h_{H P_{i}-d i-2} & \cdots & h_{H P_{i}-d i-N_{C i}}
\end{array}\right)
\end{aligned}
$$

Where:

$$
y_{i}^{*}(k)=\frac{y_{i}(k)}{C_{i}\left(z^{-1}\right)}, \Delta u_{i}^{*}(k-1)=\frac{\Delta u_{i}(k-1)}{C_{i}\left(z^{-1}\right)},
$$


And

$$
\left\{\begin{array}{l}
\operatorname{DIM}\left(\hat{G}_{i}\right)=\left(\mathrm{HP}_{i}-\mathrm{d}_{i}, \mathrm{na}_{i}+\mathrm{d}_{i}-1\right), \\
\operatorname{DIM}\left(\hat{R}_{i}\right)=\left(\mathrm{HP}_{i}-\mathrm{d}_{i}, \mathrm{nb}_{i}+\mathrm{d}_{i}-1\right), \\
\operatorname{DIM}\left(\hat{H}_{i}\right)=\left(\mathrm{HP}_{i}-\mathrm{d}_{i}, \mathrm{~N}_{C i}\right),
\end{array}\right.
$$

Denote the dimension of $\hat{G}_{i}, \hat{R}_{i}$ and $\hat{H}_{i}$, respectively.

\subsection{Law Order}

We write the criterion $\mathrm{J}$ in matrix form

$$
\left.J_{i}=\hat{Y}(k)-Y_{G}(k)\right]^{T} \chi_{i}\left[\hat{Y}(k)-Y_{G}(k)\right]+\lambda_{i} \Delta U_{i}(k)^{T} \Delta U_{i}(k)
$$

With:

$$
Y_{C i}=\left[y_{C_{i}}\left(k+d_{i}\right) \cdots y_{C i}\left(k+H P_{i}+d_{i}\right)\right]
$$

The optimal vector $\Delta U_{i}$ is:

$$
\Delta U_{i}=\left(\hat{H}_{i}^{T} \chi_{i} \hat{H}_{i}+\lambda_{u i} I_{N_{C i}}\right)^{-1} \hat{H}_{i}^{T} \hat{Y}_{i}^{T}
$$

The optimal control law is derived from analytical minimization of the previous cost function. Only the first control value is finally applied to the system.

$$
u_{i}(k)=\Delta u_{i}(k)+u_{i}(k-1)
$$

Which $\Delta u_{i}(k)$ is the first element of the vector $\Delta U_{i}$ and $I_{N_{C}}$ is diagonal matrix of size $N_{C i} * N_{C i}$ and $\chi_{i}$ is diagonal matrix of size $H P_{i}-d_{i} * H P_{i}-d_{i}$

$$
I_{N_{C i}}=\left(\begin{array}{lll}
1 & & 0 \\
& \ddots & \\
0 & & 1
\end{array}\right), \chi_{i}=\left(\begin{array}{lll}
\lambda_{y i} & & 0 \\
& \ddots & \\
0 & & \lambda_{y i}
\end{array}\right)
$$

\subsection{Constraints Formulation}

Generally, the constraints imposed on the control signal and its increment are described by inequalities forms

$$
\begin{aligned}
& u_{\min } \leq u_{i}(k) \leq u_{\max } \\
& -s u_{i} \leq \Delta u_{i}(k) \leq s u_{i}
\end{aligned}
$$

Where $u_{\min }, u_{\max }$ and $s u_{i}$ are respectively lower threshold, the upper threshold and derivative threshold of the control inputs. On the horizon controller $H C_{i}$ can be written: 


$$
\begin{aligned}
-s u_{i} & \leq \Delta u_{i}(k) \leq s u_{i}, \\
-s u_{i} & \leq \Delta u_{i}(k+1) \leq s u_{i}, \\
& \vdots \\
-s u_{i} & \leq \Delta u_{i}\left(k+H C_{i}-1\right) \leq s u_{i},
\end{aligned}
$$

Or in the condensed form:

$$
\left[\begin{array}{c}
I \\
-I
\end{array}\right] \Delta U_{i} \geq\left[\begin{array}{c}
-\beta_{1} \\
-\beta_{1}
\end{array}\right]
$$

With I is identity matrix of dimension $(\mathrm{HCi}, \mathrm{HCi}), \Delta U_{i}$ and $\beta$ are two vectors with the same dimensions $\mathrm{HCi}$ :

$$
\begin{aligned}
& \beta_{1}^{T}=\left[s u_{i} \cdots \cdots s u_{i}\right] \\
& \Delta U_{i}^{T}=\left[\Delta u_{i}(k), \cdots \cdots, \Delta u_{i}\left(k+H C_{i}-1\right)\right]
\end{aligned}
$$

On the same horizon control we obtained:

$$
\begin{gathered}
u_{\min }-u_{i}(k-1) \leq \Delta u_{i}(k) \leq u_{\max }-u_{i}(k-1), \\
u_{\min }-u_{i}(k-1) \leq \Delta u_{i}(k)+\Delta u_{i}(k+1) \leq u_{\max }-u_{i}(k-1), \\
\vdots \\
u_{\min }-u_{i}(k-1) \leq \Delta u_{i}(k)+\cdot \Delta_{i}\left(k+H C_{i}-1\right) \leq u_{\max }-u_{i}(k-1),
\end{gathered}
$$

Or in the condensed form:

$$
\left[\begin{array}{c}
W \\
-W
\end{array}\right] \Delta U_{i} \geq\left[\begin{array}{c}
\beta_{2}(k-1) \\
-\beta_{3}(k-1)
\end{array}\right],
$$

With $\beta_{2}{ }^{T}$ et $\beta_{3}{ }^{T}$ are two vectors with the same dimensions $\mathrm{HCi}$ :

$$
\begin{aligned}
& \beta_{2}^{T}(k-1)=\left[\left[u_{\min }-u_{i}(k-1)\right] \cdots \cdot\left[u_{\min }-u_{i}(k-1)\right]\right], \\
& \beta_{3}^{T}(k-1)=\left[\left[u_{\max }-u_{i}(k-1)\right], \cdots \cdot\left[u_{\max }-u_{i}(k-1)\right]\right] .
\end{aligned}
$$

And $\mathrm{W}$ is a matrix of dimension $(\mathrm{HCi}, \mathrm{HCi})$.

$$
W=\left[\begin{array}{cccc}
1 & 0 & \cdots & 0 \\
1 & 1 & 0 & 0 \\
\vdots & \vdots & \vdots & \vdots \\
1 & 1 & \cdots & 1
\end{array}\right]
$$

We can rewrite the two inequalities (3.25) and (3.28)

$$
\left[\begin{array}{l}
I \\
-I \\
W \\
-W
\end{array}\right] \Delta U_{i} \geq\left[\begin{array}{c}
-\beta_{1} \\
-\beta_{1} \\
\beta_{2}(k-1) \\
-\beta_{3}(k-1)
\end{array}\right]
$$


The problem of minimization of the criterion $\mathrm{J}$ with constraints is writing:

$$
\begin{aligned}
& \min _{\Delta \mathrm{U}_{\mathrm{i}}} \mathrm{J}_{\mathrm{i}}=\left[\hat{\mathrm{Y}}_{\mathrm{i}}(\mathrm{k})-\mathrm{Y}_{\mathrm{C}_{\mathrm{i}}}(\mathrm{k})\right]^{\mathrm{T}} \chi_{\mathrm{i}}\left[\hat{\mathrm{Y}_{\mathrm{i}}}(\mathrm{k})-\mathrm{Y}_{\mathrm{C}_{\mathrm{i}}}(\mathrm{k})\right]+\lambda_{\text {ui }} \Delta \mathrm{U}_{\mathrm{i}}(\mathrm{k})^{\mathrm{T}} \Delta \mathrm{U}_{\mathrm{i}}(\mathrm{k}) \\
& \psi \Delta U_{i} \geq \phi(k),
\end{aligned}
$$

With:

$$
\begin{aligned}
& \psi^{T}=\left[\begin{array}{llll}
I, & -I, & W^{T}, & -W^{T}
\end{array}\right]
\end{aligned}
$$

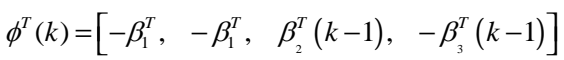

The minimization of this criterion cannot be performed analytically. The synthesis of the control law amounts to solving an optimization problem of a quadratic criterion under constraints like inequalities. During the simulations proposed subsequently, the determination of the optimal control sequence is done using the function "fmincon" of Matlab ${ }^{\mathrm{TM}}$.

\section{Decoupling Design of Generalized Predictive Control}

\subsection{Adjusting the weighting factor synchronization to the reference signal change}

In order to present the decoupling approach proposed, the TITO system presented in Fig 2 is used:

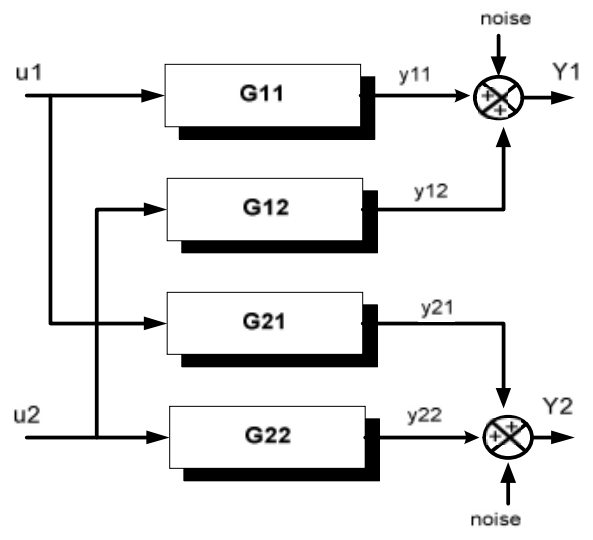

Figure 2. TITO process model.

Assume the process to be controlled is a double-input/double-outputs system .The transfer functions of the various subsystems are described by:

$$
y_{i j}(k)=\frac{z^{-d i j} B_{i j}\left(z^{-1}\right)}{A_{i j}\left(z^{-1}\right)} u_{i}(k-1)
$$

The two output variables $\left(y_{1}, y_{2}\right)$ of the process become the controlled variables, the two variable inputs $\left(u_{1}, u_{2}\right)$ are the manipulated variables and $\xi_{1}, \xi_{2}$ are non-measurable disturbances. 
$A\left(z^{-1}\right) Y(k)=B\left(z^{-1}\right) U(k-1)+\xi(k)$,

$A\left(z^{-1}\right)=\left[\begin{array}{cc}1-1.4 z^{-1}+0.45 z^{-1} & 0 \\ 0 & 1-1.2 z^{-1}+0.32 z^{-1}\end{array}\right]$,

$B\left(z^{-1}\right)=\left[\begin{array}{cc}0.209 z^{-1}+z^{-2} & 0.213-0.25 z^{-1} \\ 0.226 z^{-1}+0.324 z^{-2} & 0.131+0.109 z^{-1}\end{array}\right]$

$\xi=\left[\begin{array}{l}\xi_{1} \\ \xi_{2}\end{array}\right], U=\left[\begin{array}{l}u_{1} \\ u_{2}\end{array}\right], Y=\left[\begin{array}{l}y_{1} \\ y_{2}\end{array}\right]$

We write the criterion $\mathbf{J}$ of each controller

GPC1:

$$
J_{1}=\lambda_{y 1} \sum_{j=N_{1}}^{H P_{1}}\left[y_{C_{1}}(k+t)-\hat{y}_{1}(k+t)\right]^{2}+\lambda_{u 1} \sum_{j=1}^{N_{C}} \Delta u_{1}^{2}(k+t-1),
$$

GPC2:

$$
J_{2}=\lambda_{y 2} \sum_{j=N_{2}}^{H P_{2}}\left[y_{C_{2}}(k+t)-\hat{y}_{2}(k+t)\right]^{2}+\lambda_{u 2} \sum_{j=1}^{N_{C}} \Delta u_{2}^{2}(k+t-1)
$$

The main idea of decoupling controller is the following [3]: when some reference changes its value (for example: set-point of y1) controller firstly increases only $\lambda_{y 2}$ in the second loop, and then calculate optimal control vector (32) and (33). This control vector would minimize output deviation caused by reference changes. Values $\lambda_{y i}$ are evaluated from equation:

$\lambda_{\mathrm{yi}}=1+\sum_{\mathrm{j}=1}^{\mathrm{m}} \frac{\mathrm{K}_{\mathrm{ri}}}{\mathrm{M}\left(\mathrm{q}^{-1}\right)} \Delta \mathrm{y}_{\mathrm{Cj}}(\mathrm{k})$

$\mathrm{K}_{\mathrm{ri}}$ : maximum error weight in $\mathrm{j}$ loop,

$\Delta \mathrm{y}_{\mathrm{Cj}}:$ reference change on $\mathrm{j}$ input,

$\mathrm{M}\left(\mathrm{q}^{-1}\right)$ : Polynomial of $\mathrm{q}^{-1}$ defined by designer.

Results of simulations in which we take into count the change of the set point and the effect of disturbances of showed the limit of this method.

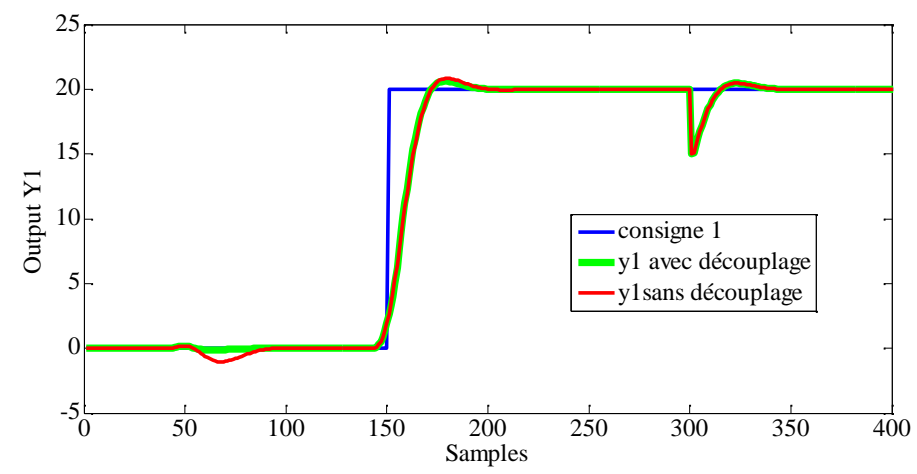

Figure 3. Evolution of y1 with tuning weighting factor by reference change 
International Journal of Information Technology, Control and Automation (IJITCA) Vol.2, No.3, July 2012

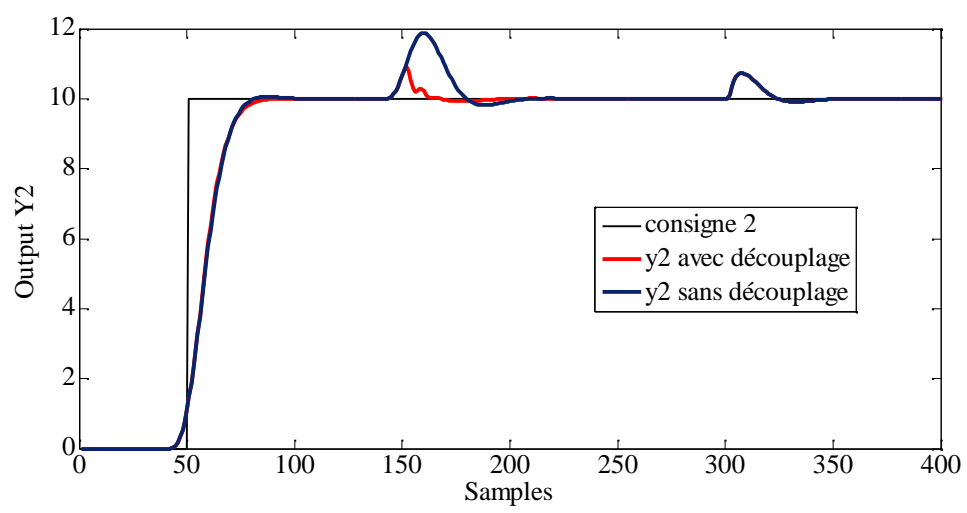

Figure 4. Evolution of y2 with tuning weighting factor by reference change

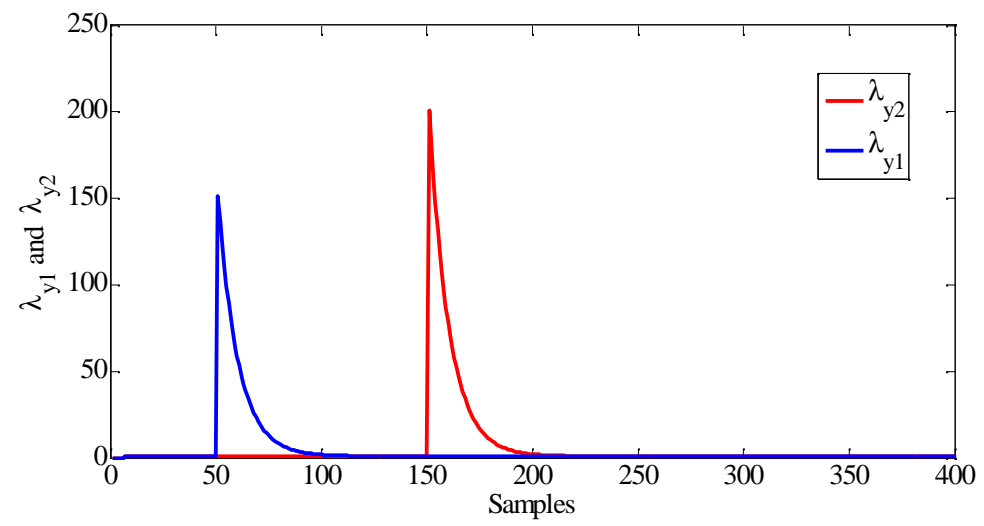

Figure 5. Evolution of the weighting factor $\lambda_{y 1}$ and $\lambda_{y 2}$.

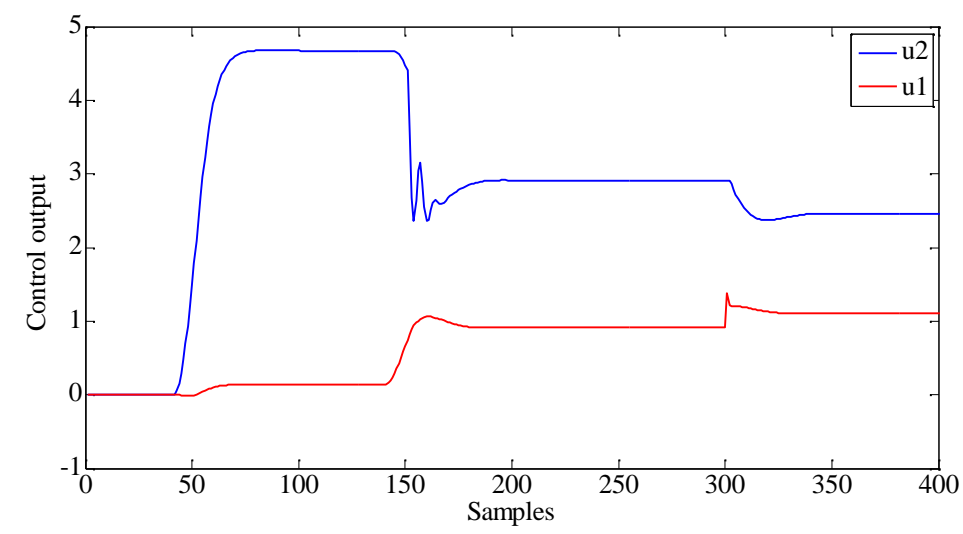

Figure 6. Evolution of the control output $\mathrm{u} 1$ and $\mathrm{u} 2$

The method of decoupling cannot reduce the coupling effect caused by external disturbance. To resolve this problem, we propose an adaptive weighting factor dependent on the actual control error. 


\subsection{Tuning weighting factor by the error observation}

To overcome the drawback of the previous method we present a new approach to decoupling the structure of the decoupling control is also described in the figure 7.

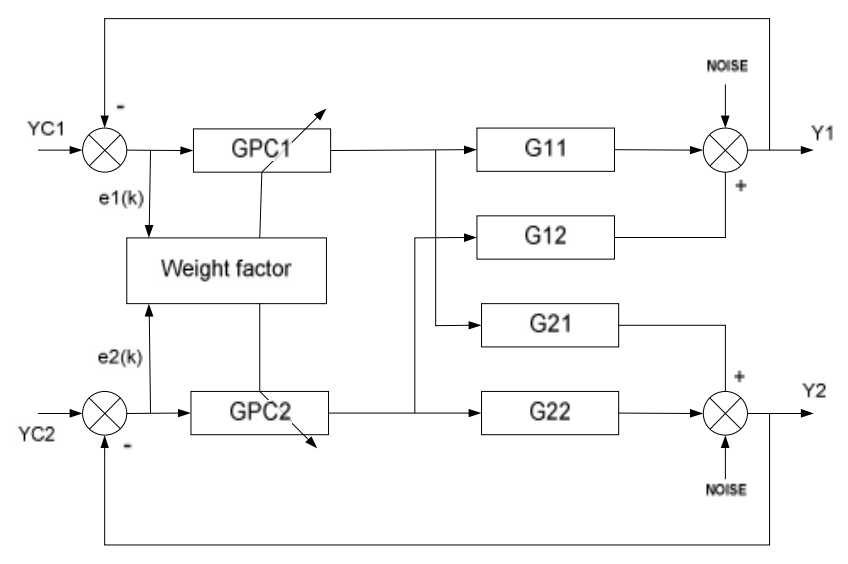

Figure 7. Control structure of generalized predictive control with weighting factor adjusting.

The main idea of decoupling controller is the following: The error weight factor of the output variable whose reference is kept constant should be increased and synchronized with the variable whose reference is modified. We note that a high value of the weighting factor $\lambda_{\max }$ on output error can generate a very drastic change in controlled variables and degrades the control performance, what we are trying to avoid in most cases. On the other hand, a low value of weighting factor $\lambda_{\min }$ does not allow reducing the effect of the coupling caused by the other variable.

To satisfy some performance (smooth control movements and minimum coupling effect), we establish the weighting factors expressions

$$
\begin{aligned}
& \lambda_{y 1}=\left\{\begin{array}{cc}
\lambda_{\max 1} & \lambda_{y 1} \geq \lambda_{\max 1} \\
\lambda_{\min 1}+\exp \left(\left|\varepsilon_{2}(k)\right|\right) & \lambda_{y 1} \leq \lambda_{\max 1}
\end{array}\right\}, \\
& \lambda_{y 2}=\left\{\begin{array}{cc}
\lambda_{\max 2} & \lambda_{y 2} \geq \lambda_{\max 2} \\
\lambda_{\min 2}+\exp \left(\left|\varepsilon_{1}(k)\right|\right) & \lambda_{y 2} \leq \lambda_{\max 2}
\end{array}\right\} .
\end{aligned}
$$

With: $\varepsilon_{1}(k)=y_{C_{1}}(k)-y_{1}(k), \quad \varepsilon_{2}(k)=y_{C_{2}}(k)-y_{2}(k)$.

A good decoupling with better control performance seemed to be optimal when an exponential change of the weighting factors is used and the limit value is respected. Tuning of $\lambda_{y_{i}}$ depends on process parameters [7] and can vary in broad range [1, 1000]. Higher $\lambda_{\max }$ leads to better decoupling. To check the validation of decoupling designed a simulation results are presented in Figs 8 and 9. It should be mentioned that control horizon is $N_{G_{1}}=N_{C_{2}}=1$ and the control prediction is $H P_{1}=H P_{2}=15$. Weighting of the control error for the first and second manipulated variables are: 
International Journal of Information Technology, Control and Automation (IJITCA) Vol.2, No.3, July 2012

$$
\begin{aligned}
& \lambda_{y 1}=\left\{\begin{array}{cc}
50 & \lambda_{y 1} \geq 50 \\
1+\exp \left(\left|\varepsilon_{2}(k)\right|\right) & \lambda_{y 1} \leq 50
\end{array}\right\}, \\
& \lambda_{y 2}=\left\{\begin{array}{cc}
50 & \lambda_{y 2} \geq 50 \\
1+\exp \left(\left|\varepsilon_{1}(k)\right|\right) & \lambda_{y 2} \leq 50
\end{array}\right\} .
\end{aligned}
$$

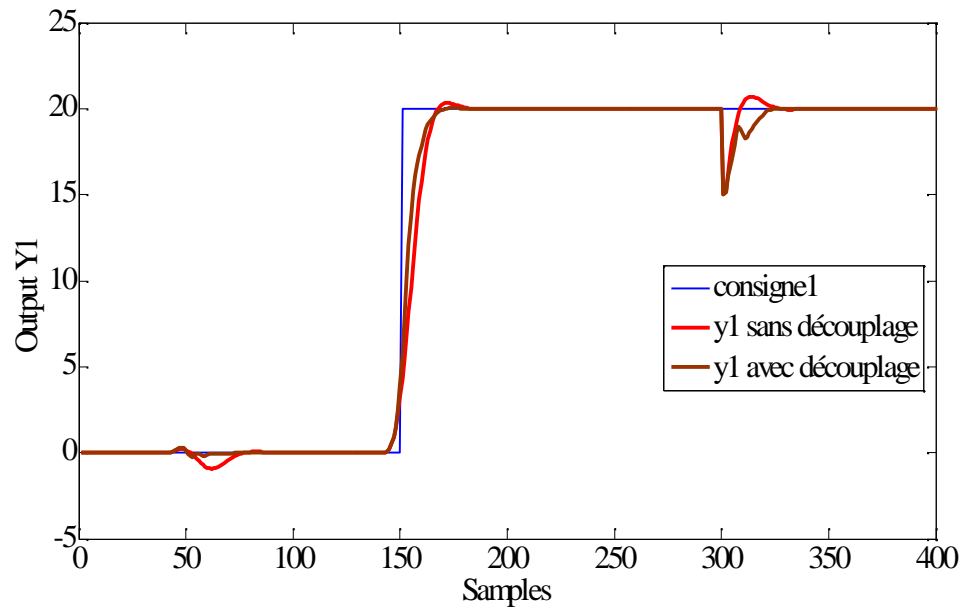

Figure 8. Evolution of y1 with tuning weighting factor by error observation and without decoupling

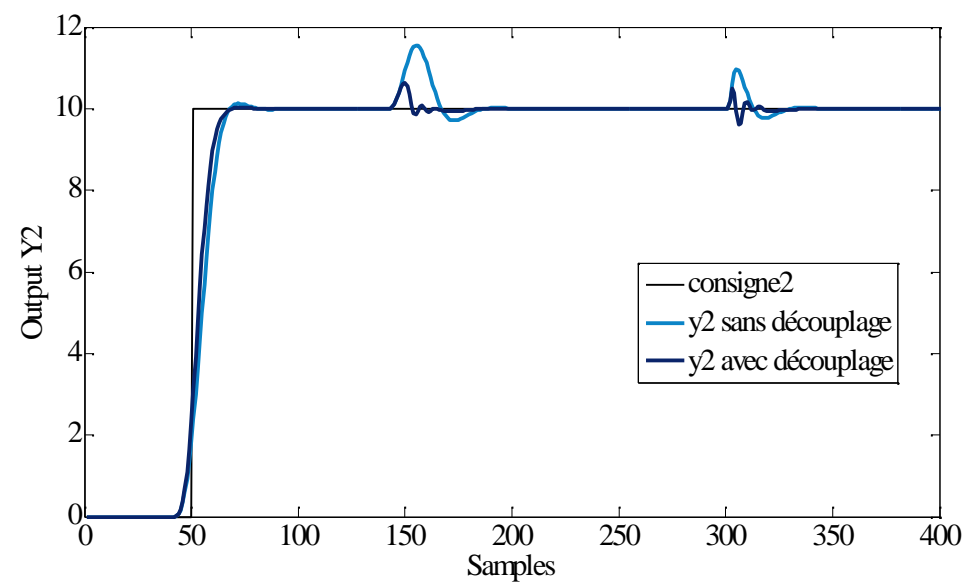

Figure 9. Evolution of $\mathrm{y} 2$ with tuning weighting factor by error observation and without decoupling 
International Journal of Information Technology, Control and Automation (IJITCA) Vol.2, No.3, July 2012

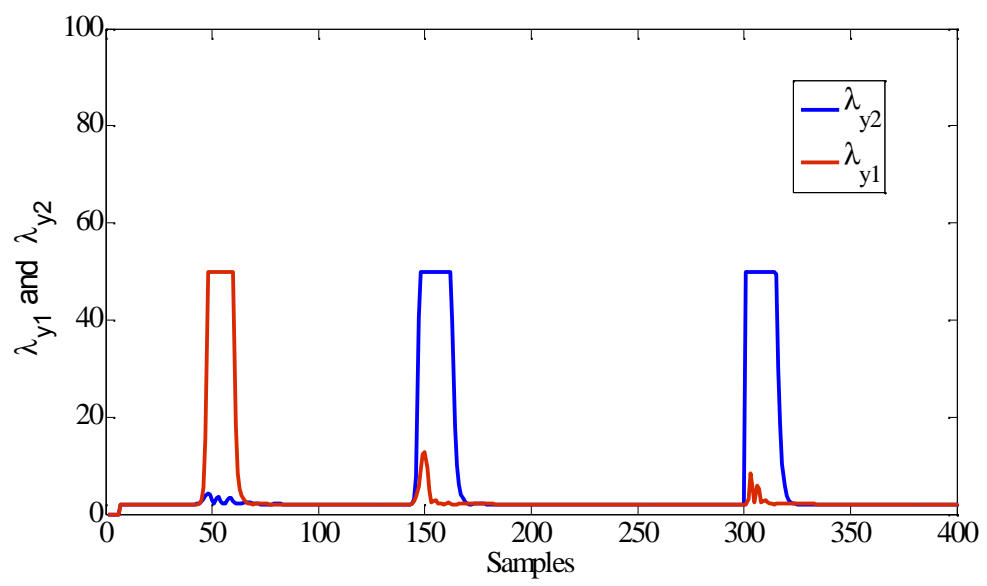

Figure 10. Evolution of. the tuning weighting factor $\lambda_{y 1}$ and $\lambda_{y 2}$ by error observation

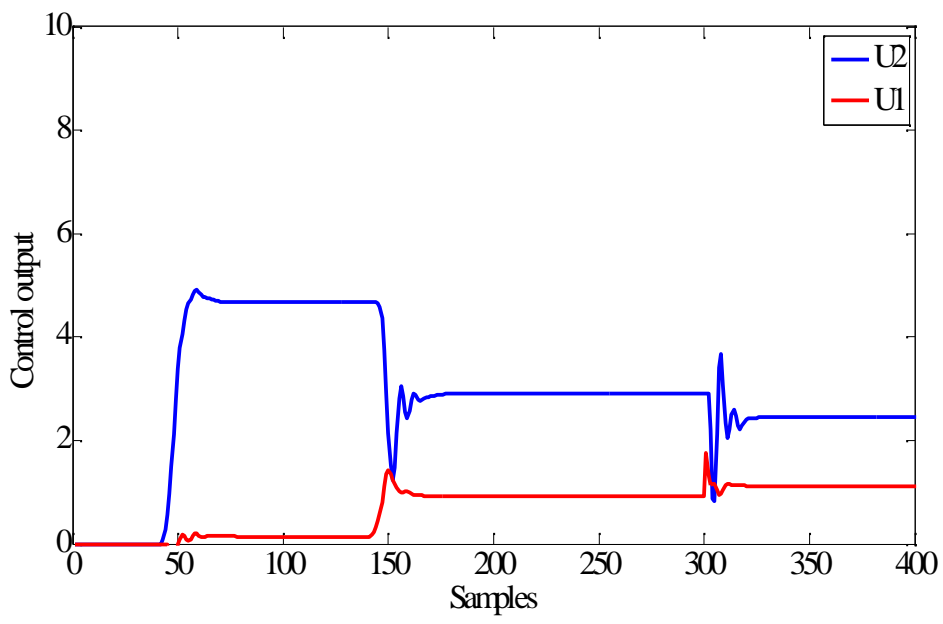

Figure 11. Evolution of the control output $\mathrm{u} 1$ and $\mathrm{u} 2$

Fig 10 shows that the weighting factor dependent on the actual output error which allow at the same time to be powerful in dynamic and static decoupling. Fig 11 shows the evolution of the control signal of y1 and y2.

The disadvantage of this method is when the controlled variable is subjected to a disturbance. This disturbance affects the second variable without any adaptive weights factor error. To resolve this problem, the weighting factor should always be dependent on the actual control error.

\section{APPLiCATION TO AN INCUBATOR SYSTEM}

This section emphasizes on examining the performance of the proposed Decoupling Generalized Predictive Controller (DGPC) for temperature and humidity control of a new born incubator system. 


\subsection{Experiment setup}

A neonatal incubator is, usually, a small (approximately: $0.5 \times 0.5 \times 1 \mathrm{~m}^{3}$ ) cabinet with transparent walls so that the infant can be easily observed.

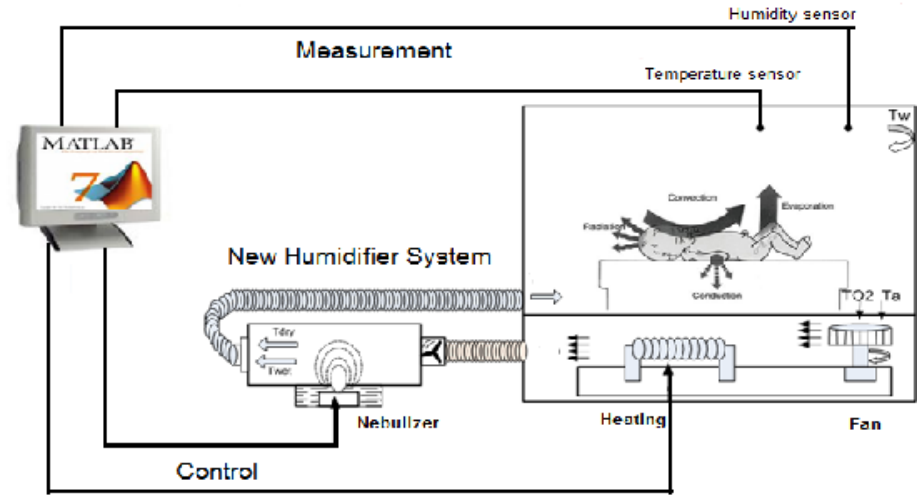

Figure12. Schematic of the incubator process with experimental arrangement for active humidification to control temperature and Humidity.

The device includes an AC-powered heater, a fan to circulate the warmed air, a container for water to add humidity and access ports for nursing care. The passive humidifier cannot provide a high humidity level at low temperature such as in the range of $\left[20^{\circ} \mathrm{C}\right.$ to $\left.38^{\circ} \mathrm{C}\right]$. So, we replaced the passive humidifier by an external block based on ultrasonic nebulizer system which is an instrument for converting a liquid into a fine spray. The active humidification system is able to increase the humidity to $80 \%$. Also we developed a microcontroller-based system devoted to control the humidity and the heating of the new born incubator. The system developed measures the air temperature and the humidity by two sensors LM35 and SY-230. Then, these data are exported to a microcomputer to be analyzed and to develop a model for the incubator [14],[13]. The aim of this study is to design and implement a closed loop control system to regulate the temperature and humidity inside a neonatal incubator which presented in Fig 12. The presence of coupling makes it impossible to set one without affecting the other. To eliminate the couplings between temperature and humidity, we propose a multivariable decoupled control by tuning the weighting factor.

\subsection{Model of the incubator process}

Before proceeding with the real-time control, it is necessary to pass through the modeling system. For this reason, an experimental method is proposed for modeling of the TITO system. The incubator system has Two-Inputs and Two-Outputs.

The inputs to the system are

$u_{1}$ : control signal applied to the heater,

$u_{2}$ : control signal applied to the nebulizer.

The outputs are:

$y_{1}$ : temperature value output signal,

$y_{2}$ : humidity level output signal. 
We define each sub system by Auto Regressive Integrated Moving Average with eXternal inputs, (ARIMAX) model [1].

$$
y_{i j}(k)=z^{-d i j} \frac{B_{i j}\left(z^{-1}\right)}{A_{i j}\left(z^{-1}\right)} u_{i}(k-1)+\frac{C_{i}\left(z^{-1}\right)}{A_{i j}\left(z^{-1}\right) \Delta\left(z^{-1}\right)} e_{i}(k) .
$$

Where $d_{i j}$ is time delay, $e_{i}$ is white noise and $A_{i j}, B_{i j}, C_{i}$ are:

$$
\begin{aligned}
& A_{i j}\left(z^{-1}\right)=1+a_{1} z^{-1}+a_{2} z^{-2}+\cdots+a_{n a} z^{-n a}, \\
& B_{i j}\left(z^{-1}\right)=b_{0}+b_{1} z^{-1}+b_{2} z^{-2}+\cdots+b_{n b} z^{-n b}, \\
& C_{i}\left(z^{-1}\right)=1+c_{1} z^{-1}+c_{2} z^{-2}+\cdots+c_{n c} z^{-n c} .
\end{aligned}
$$

We present the multivariable system as matrix form:

$$
\begin{aligned}
{\left[\begin{array}{l}
y_{1}(k) \\
y_{2}(k)
\end{array}\right]=\left[\begin{array}{ll}
G_{11}(z) & G_{12}(z) \\
G_{21}(z) & G_{22}(z)
\end{array}\right]\left[\begin{array}{l}
u_{1}(k) \\
u_{2}(k)
\end{array}\right]+\left[\begin{array}{l}
\xi \\
\xi_{2}
\end{array}\right] . } \\
G(z)=\left[\begin{array}{ll}
G_{11} & G_{12} \\
G_{21} & G_{22}
\end{array}\right]=\left[\begin{array}{ll}
z^{-d 11} \frac{B_{11}\left(z^{-1}\right)}{A_{11}\left(z^{-1}\right)} & z^{-d 12} \frac{B_{12}\left(z^{-1}\right)}{A_{11}\left(z^{-1}\right)} \\
z^{-d 21} \frac{B_{21}\left(z^{-1}\right)}{A_{21}\left(z^{-1}\right)} & z^{-d 22} \frac{B_{22}\left(z^{-1}\right)}{A_{22}\left(z^{-1}\right)}
\end{array}\right] \\
= \\
\left.\begin{array}{ll}
\frac{z^{-10}\left(5.9746 \mathrm{e}-004+5.9412 \mathrm{e}-004 z^{-1}\right)}{1-0.3467 z^{-1}-0.6463 \mathrm{z}^{-2}} & 0 \\
\frac{\mathrm{z}^{-32}\left(1.3698 \mathrm{e}-004-4.1930 \mathrm{e}-004 \mathrm{z}^{-1}\right)}{1-0.28321 z^{-1}-0.7133 z^{-2}} & \frac{\mathrm{z}^{-3}\left(0.00203+0.00088 \mathrm{z}^{-1}\right)}{1-0.5091 z^{-1}-0.4262 z^{-2}}
\end{array}\right] .
\end{aligned}
$$

\subsection{Computer simulations and discussions}

Before proceeding with the real time control, it is important to conduct computer simulation with Matlab software to check the feasibility of the proposed approach control. The principal parameters are set as follows: The prediction horizons are $H P_{1}=40, H P_{2}=20$, the control horizons are $N c_{1}=N c_{2}=1$ and the weighting factors of the control increments are $\lambda_{11}=0242, \lambda_{12}=04712$.

The coupling effect was suppressed by the method of changing the weighting factor as a function of the control error. A good decoupling could be achieved by the same dependence of the weighting factors as in equation (39) with

$$
\lambda_{y 1}=1 \text { and } \lambda_{y 2}=\left\{\begin{array}{cc}
5 & \lambda_{y 2} \geq 50 \\
\lambda_{y 2 \min }+\exp \left(\left|\varepsilon_{1}(k)\right|\right) & \lambda_{y 2} \leq 50
\end{array}\right\}
$$

In Fig 17, we obtained the ranges of the control signal applied to the heater and to the nebulizer that scaled between $0 \%$ and $100 \%$. The multivariable control is simulated with constraints and at sampling times $\mathrm{T}=20$ seconds.

Chosen the inputs constraints:

$$
\begin{aligned}
& 0 \% \leq u_{1} \leq 100 \%, \\
& 0 \% \leq u_{2} \leq 100 \% .
\end{aligned}
$$

The described model (43) has strong interaction between input u1 and output y2. For this reason, the analyze has been done by changing temperature's reference and observing output signal of the humidity. Tow method are used and compared: tuning the weighting factor $\lambda_{v 2}$ with error 
observation and with synchronization to set point change. For $\lambda_{\mathrm{y} 1}$, it remains constant and justified by the low interaction between $u_{2}$ and $G_{11}$.

In Fig 13, we present the simulation result of set-point tracking temperature which increases from initial temperature to $25^{\circ} \mathrm{C}$ then to $28^{\circ} \mathrm{C}$ and decreases to $26^{\circ} \mathrm{C}$. In Fig 14, it can be noticed that, there are great differences in the R.H. (Relative Humidity) response due to the choice of the error weighting factor. It is clear that adaptive weighting factor with reference change does not reduce the effect of coupling due to external perturbation. But decoupling with adaptive weight factor that dependent on the actual control error seems a good method to reduce the coupling effect due to the references change or external perturbation. In Fig 15, we present the evolution of the weighting factor with error output. We observe that the weight is very sensitive to the output error. To reduce this sensitivity, we applied a first order filter. In Fig 16, we present the evolution of the weighting factor which is synchronized with reference change. The adaptive error weighting factor is synchronized to the reference signal change. In Fig. 17, we observed that an increase of $\lambda_{y 2}$ generates a very drastic change in the manipulated variable (humidity control) which is an indicator of the decoupling effects. The advantage of using an adaptive error weighting factor is reducing the coupling effect cause by set point change and external perturbation.

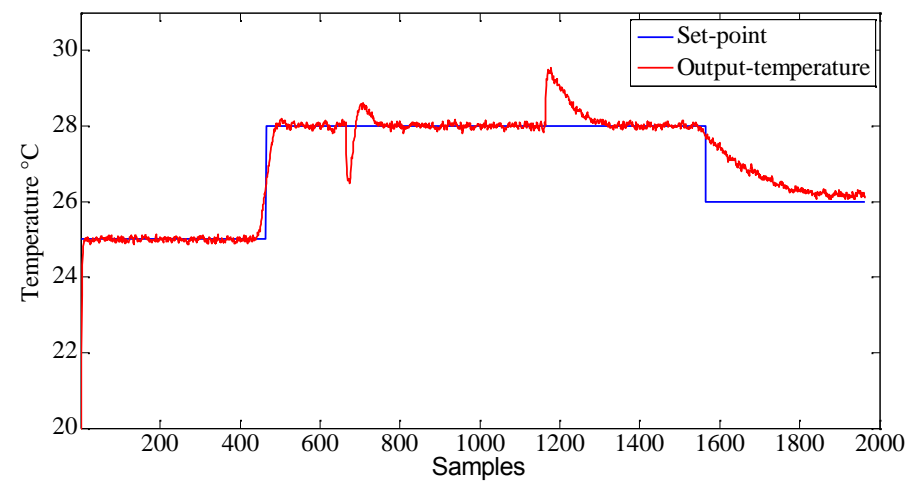

Figure 13. Simulation result of set-point tracking temperature for predictive decoupling control.

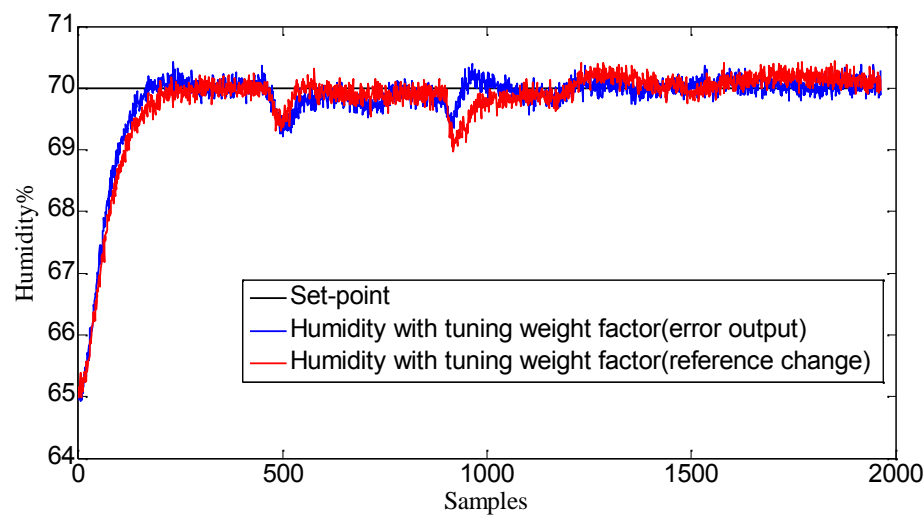

Figure 14. Compared result of set-point tracking humidity with tuning weight factor by reference change and tuning weight factor by error output observation. 
International Journal of Information Technology, Control and Automation (IJITCA) Vol.2, No.3, July 2012

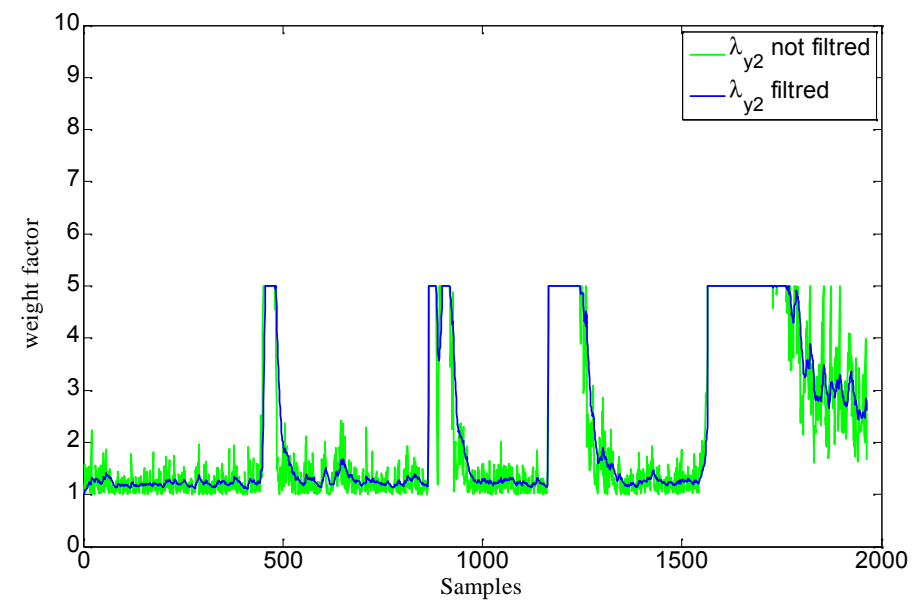

Figure 15. Evolution of the filtered weighting factor $\lambda_{y 2}$.

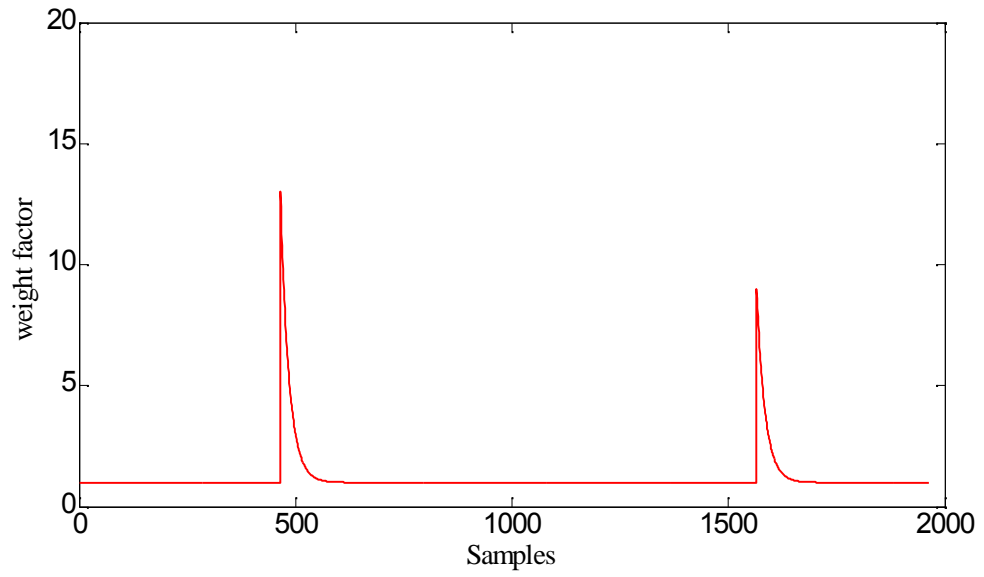

Figure16. Evolution of the weighting factor synchronized with change set point.

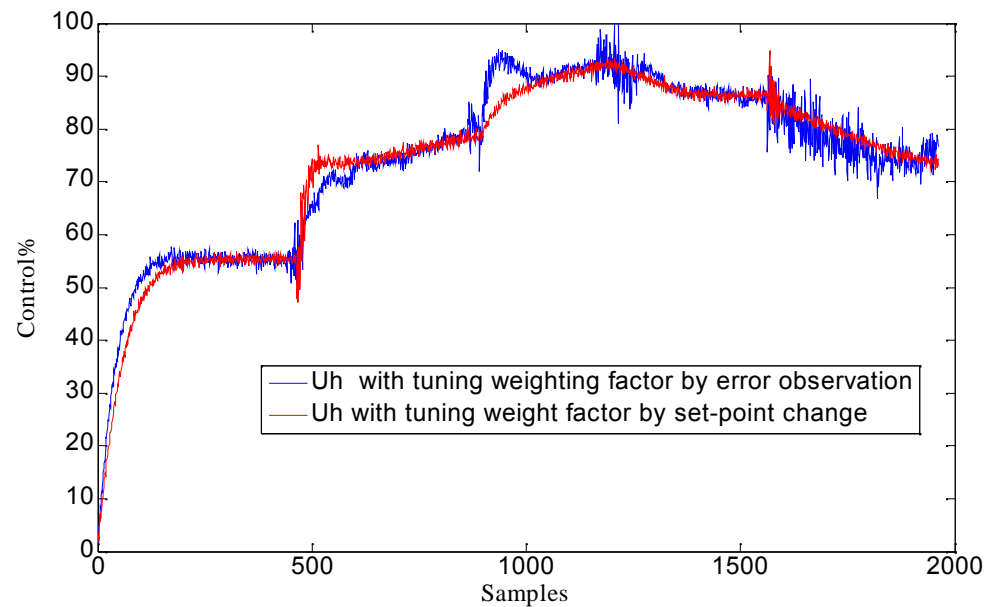

Figure 17. Compared results of decoupling control humidity by set-point and error output tuning weighting factor. 


\section{CONCLuSions}

This paper has developed a systematic design approach for Decoupling Generalized Predictive temperature and humidity Control (DGPC) of an incubator process. Simulations results have been demonstrated that decoupling by tuning weighting factor with synchronization in set-point change does not allow a good decoupling relative to the tuning error observation. Proposed concept of adaptive weighting leads to simple decoupling controller and it is not applicable only to predictive control but to all optimal multivariable controllers based on some cost function minimization. The simulation and experimental results have shown that the proposed control method is capable of giving satisfactory temperature and humidity control performance for the incubator system.

\section{REFERENCES}

[1] A. K Abbas and S. Leonhardt, (2009) "System Identification of neonatal incubator based on adaptive ARMAX technique", IFMBE Proc, vol.22, pp.2515-2519.

[2] A. Ogunnaike and W. H. Ray, (1994) "Process Dynamics, Modeling, and Control", Oxford Univ. Press.

[3] Bego, O. Peric, N. I. Petrovic, (2000) "Decoupling multivariable GPC with reference observation", 10th Mediterranean Electrotechnical Conference, vol.2, pp.819 - 822.

[4] C. C. Tsai and C. H. Huang, "Model reference adaptive predi- ctive control for a variable-frequency oil-cooling machine", IEEE Trans. Ind. Electron, 51,vol. 2, pp. 330-339, 2004.

[5] D. W. Clarke, C. Mohtadi and P. S. Tuffs, (1987) "Generalized Predictive Control - Part I and II", Automatica, 23(2), pp. 137-160.

[6] D. W. Clarke, (1988) "Application of Generalized Predictive Control to Industrial Processes", IEEE Control System Magazine, vol. 8, pp.49-55.

[7] F. Arousi, (2009) "Predictive Control Algorithms for Linear and non Linear Processes", Thesis,.

[8] F. Telliez, V. Bach, S. Delanaud, H. M. Baye, A. Leke, A. Apdoh and M. Abidiche, (1999) "Influence du niveau d'humidité de l'air sur le sommeil du nouveau-né en incubateur", RBM. Revue européenne de biotechnologie médicale, vol.21, pp.171-176.

[9] Gler and M. Burunkaya, (2002) "Humidity control of an incubator using the microcontroller-based active humidifier system employing an ultrasonic nebulizer", Journal of Medical Engineering and Technology, vol.26, pp.82-88.

[10] G. Li, D. P. Stoten and J.-Y. Tu, (2010) "Model predictive control of dynamically substructured systems with application to a servohydraulically actuated mechanical plant", IET Control Theory Appl., 4(2), pp. 253-264.

[11] J. Richalet, D. O’Donavan, (2009) "Elementary Predictive Functional Control", Springer Verlag, Berlin, 201-105.

[12] J. T. J. Richalet, A. Rault and J. Papon, (1978) "Model predictive heuristic control: Applications to industial processes", Automatica, vol.14, pp. 413-428.

[13] M. A. Zermani, E. Feki and A. Mami, (2011) "Application of Genetic Algorithms in identification and control of a new system humidification inside a newborn incubator", International Conference on Communications, Computing and Control Applications, pp. 1-6.

[14] M. A. Zermani, E. Feki and A. Mami, (2011) "Application of Adaptive Predictive Control to a Newborn Incubator", American J. of Engineering and Applied Sciences, 4 (2), pp. 235-243. 